\title{
Quantum Interference in Disordered Ferromagnet $\mathrm{U}_{2} \mathrm{NiSi}_{3}$
}

\author{
D. GnidA*, M. Szlawska, P. Wiśniewski And D. Kaczorowski \\ Institute of Low Temperature and Structure Research, Polish Academy of Sciences, \\ P.O. Box 1410, 50-950 Wrocław, Poland
}

\begin{abstract}
A single-crystalline sample of disordered ferromagnetic $\mathrm{U}_{2} \mathrm{NiSi}_{3}$ was investigated by means of electrical resistivity measurements under ambient and high hydrostatic pressure. Temperature dependences of the electrical resistivity clearly reveal interplay of the ferromagnetic ordering and quantum interference effects resulting from crystallographic disorder. Electron-electron interaction manifests itself as a $T^{0.5}$ increase in the in-plane and out-of-plane electrical resistivity below $5 \mathrm{~K}$. Weak localization is observed solely in the $a b$-plane as a linear-in- $T$ contribution to resistivity, which suggests that internal magnetic field does not break the interference of scattered electron waves in $a b$-plane. Applied hydrostatic pressure does not affect the $T^{0.5}$ electron-electron interaction contribution, however it diminishes the impact of weak localization on the $a b$-plane resistivity.
\end{abstract}

DOI: 10.12693/APhysPolA.127.451

PACS: 72.15.-v, 75.50.Lk, 72.15.Rn

\section{Introduction}

It is well known that increase in amount of impurities in simple metallic compounds leads not only to increase in residual resistivity but may also result in formation of a low temperature minimum in the electrical resistivity. The origin of this phenomenon was theoretically explained in late seventies [1, 2]. Distinct deviation from the Boltzmann theory has been found in the resistivity behaviour of disordered conductors, which occurs due to interferences between scattered partial electron waves. In order to compensate for that deviation a correction has been introduced that accounts for coherent superposition of wave functions of elastically scattered electrons, called the weak localisation. Such correction is significant if electrons experience multiple elastic scattering on impurities before the constructive interference is destroyed in an inelastic scattering process [2-4]. In addition to that mechanism, electrons moving in a disordered potential may also interact with fluctuating inhomogeneous local electron charge density induced by other electrons, which results in electron-electron interaction correction to the conductivity with the characteristic $T^{0.5}$ dependence $[2,4]$. Though both quantum corrections are commonly observed in many disordered conductors, most of the quantitative investigations were limited to simple metallic systems with nonmagnetic ground states.

The main aim of our work was to determine the role of structural disorder in the low-temperature behaviour of the strongly anisotropic ferromagnet $\mathrm{U}_{2} \mathrm{NiSi}_{3}$. The compound crystallizes with the $\mathrm{AlB}_{2}$-type structure, in which the $\mathrm{U}$ atoms occupy the $\mathrm{Al}(\mathrm{la})$ position, while the $\mathrm{Ni}$ and Si atoms share the unique $\mathrm{B}(2 \mathrm{~d})$ site. The atomic disorder on the latter site leads to a small value of the residual resistivity ratio (RRR) close to 1 and to an enhanced

*corresponding author; e-mail: D.Gnida@int.pan.wroc.pl residual resistivity, which amounts to about $390 \mu \Omega \mathrm{cm}$ and $160 \mu \Omega \mathrm{cm}$ for the electric current flowing perpendicular and longitudinal to the $c$-axis, respectively $[5,6]$. As far as the magnetic properties are concerned, $\mathrm{U}_{2} \mathrm{NiSi}_{3}$ was characterized in the early literature reports as a spinglass system with the freezing temperature of $22 \mathrm{~K}$ [7] or a re-entrant spin-glass with the ferromagnetic transition at $25 \mathrm{~K}$ [8]. Alternatively, it was described as a ferromagnet with the Curie temperature $T_{\mathrm{C}}=26 \mathrm{~K}[9]$ or $T_{\mathrm{C}}=30 \mathrm{~K}[10]$. In the latter study, the polarized neutron diffraction measurement revealed that the uranium magnetic moments of $0.6 \mu_{\mathrm{B}}$ are oriented in the ferromagnetic state perpendicular to the $c$-axis. Most recently, thermodynamic, electrical transport and neutron diffraction measurements of single-crystalline $\mathrm{U}_{2} \mathrm{NiSi}_{3}$ confirmed the ferromagnetic ordering below $26 \mathrm{~K}$, with the uranium magnetic moments of $1.05 \mu_{\mathrm{B}}[5]$.

\section{Experimental details}

High-quality single crystal of $\mathrm{U}_{2} \mathrm{NiSi}_{3}$ was grown by the Czochralski technique in a tetra-arc furnace under ultrapure argon atmosphere. Its hexagonal $\mathrm{AlB}_{2}$-type crystal structure was confirmed on a four-circle X-ray diffractometer (KUMA Diffraction) equipped with a chargecoupled-device camera, using Mo $K_{\alpha}$ radiation.

DC magnetic measurements were performed in the temperature interval $1.72-400 \mathrm{~K}$ using a superconducting quantum interference device (SQUID) magnetometer (Quantum Design MPMS-5). The results corroborated the ferromagnetic behaviour reported in Ref. [5] and additionally verified the absence of impurity phase $\mathrm{UNiSi}_{2}$ that was observed in our previous study [6].

Four-point AC electrical resistivity measurements were performed from room temperature down to $2 \mathrm{~K}$ in hydrostatic pressures up to $2.4 \mathrm{GPa}$ using a PPMS platform (Quantum Design PPMS-9) and a high-pressure clamp cell designed for this equipment (CamCool). 


\section{Results and discussion}

Figure 1a and b presents the temperature dependences of the resistivity ratios $\rho_{c}(T) / \rho_{c}(2 \mathrm{~K})$ and $\rho_{a b}(T) / \rho_{a b}(2 \mathrm{~K})$ measured on single-crystalline $\mathrm{U}_{2} \mathrm{NiSi}_{3}$ at ambient pressure and under applied hydrostatic pressure of $2 \mathrm{GPa}$ or $2.4 \mathrm{GPa}$. As can be inferred from these figures, at ambient pressure the value of RRR amounts only to 1.06 and 0.98 for the in-plane $\rho_{a b}$ and out-ofplane $\rho_{c}$ resistivity, respectively. In the paramagnetic region, both components change with temperature in similar manner. Upon cooling from $300 \mathrm{~K}$ their values decrease to minima placed at $37 \mathrm{~K}$ and $45 \mathrm{~K}$, respectively. Further decreasing temperature down to $2 \mathrm{~K}$ causes distinct difference in the temperature evolution of the ratio $\rho(T) / \rho(2 \mathrm{~K})$. For the current parallel to the hard magnetic axis, $j \| c$, the onset of the ferromagnetic state manifests itself in a maximum at $T_{\mathrm{C}}=27.7 \mathrm{~K}$. Then, the $\rho_{c}(T) / \rho_{c}(2 \mathrm{~K})$ curve displays another minimum located near $10.7 \mathrm{~K}$. On the contrary, no clear anomaly at the magnetic phase transition is observed in the electrical resistivity measured with the current flowing within the hexagonal $a b$ plane. In this case, merely a tiny feature at $T_{\mathrm{C}}$ can be noticed in the temperature derivative of the $\rho_{a b}(T) / \rho_{a b}(2 \mathrm{~K})$ variation (see the inset to Fig. 1b).

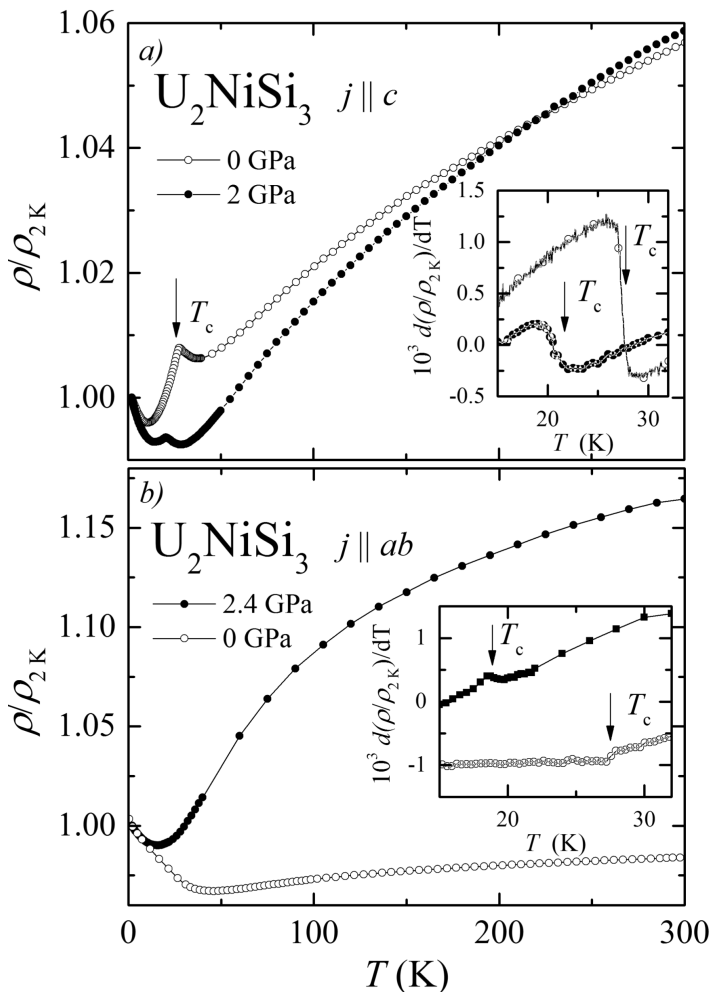

Fig. 1. Temperature dependences of the electrical resistivity ratio $\rho(T) / \rho(2 \mathrm{~K})$ of single-crystalline $\mathrm{U}_{2} \mathrm{NiSi}_{3}$ taken at ambient and applied hydrostatic pressure with the current flowing along the crystallographic $c$-axis $(a)$ and in the $a b$-plane $(b)$. The insets show the first derivatives of $\rho(T) / \rho(2 \mathrm{~K})$. The arrows indicate the Curie temperature.
From the insets to Fig. 1a and b it is also evident that under pressure the ferromagnetic phase transition shifts to about $20.4 \mathrm{~K}$ at $2 \mathrm{GPa}$ and $19 \mathrm{~K}$ at $2.4 \mathrm{GPa}$.

Simultaneously, the minima above $T_{\mathrm{C}}$ shift to $15.5 \mathrm{~K}$ for the in-plane and to $28.5 \mathrm{~K}$ for the out-of-plane resistivity components. While for the $c$-axis resistivity the effect of pressure on RRR is very small, RRR of the $a b$ plane component increases up to 1.16 at $2.4 \mathrm{GPa}$. Since hydrostatic pressure is not expected to influence elastic mean free path of carriers, the observed change in RRR seems to result from pressure-induced changes in the electronic structure of $\mathrm{U}_{2} \mathrm{NiSi}_{3}$.

Figure $2 \mathrm{a}$ and $\mathrm{b}$ presents the low-temperature $\rho(T) / \rho(2 \mathrm{~K})$ data as a function of $T^{0.5}$. Clearly, below about $5 \mathrm{~K}$, the $c$-axis resistivity can be described as $A T^{0.5}$ with $A=-2.7 \times 10^{-3} \mathrm{~K}^{-1}$ at ambient pressure and $A=-4.1 \times 10^{-3} \mathrm{~K}^{-1}$ at $2 \mathrm{GPa}$. Similar squareroot-of- $T$ dependence is also observed for the in-plane resistivity with the coefficient $A=-5 \times 10^{-3} \mathrm{~K}^{-1}$ being nearly pressure independent. Remarkably, the $a b$-plane resistivity taken at ambient is proportional to temperature in a wide temperature range $8-25 \mathrm{~K}$ (cf. the inset to Fig. 2b).

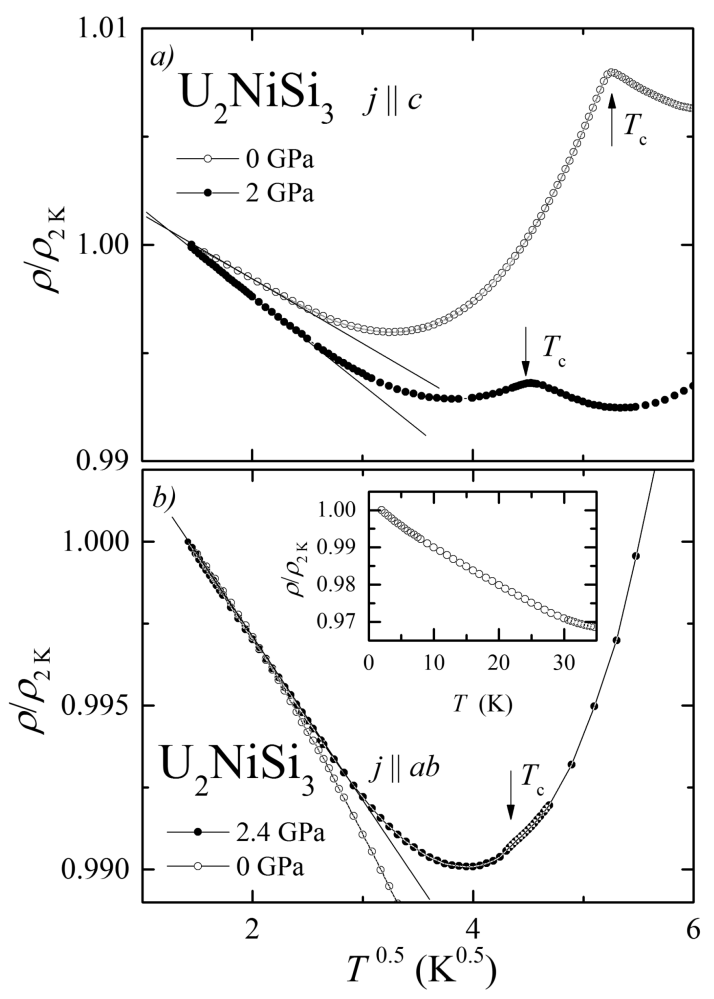

Fig. 2. Electrical resistivity ratio $\rho(T) / \rho(2 \mathrm{~K})$ of singlecrystalline $\mathrm{U}_{2} \mathrm{NiSi}_{3}$ plotted as a function of $T^{0.5}$. The data were taken at ambient pressure and under hydrostatic pressure with the current flowing along the crystallographic $c$-axis $(a)$ and in the $a b$-plane $(b)$. The arrows indicate Curie temperature. The inset shows a linear-in- $T$ dependence of the $a b$-plane resistivity. 
The observed low-temperature upturns in the $\rho(T) / \rho(2 \mathrm{~K})$ curves of $\mathrm{U}_{2} \mathrm{NiSi}_{3}$ can hardly be attributed to conventional Kondo effect, because strong internal magnetic exchange field in this ferromagnet probably prevents the spins of conduction electrons from flipping under scattering processes. In turn, it seems very unlikely that the low-temperature electrical transport in $\mathrm{U}_{2} \mathrm{NiSi}_{3}$ can be governed by nonmagnetic Kondo effect, in which conduction electrons interact with an impurity that can change its position in a double well potential. Though such interaction leads to a two-channel non-Fermi liquid behaviour characterized by a $T^{0.5}$ dependence of the resistivity, the characteristic energy scale for Kondo tunnelling was predicted to be very small and the splitting of two lowest energy levels always exceeds $T_{\mathrm{K}}$ [11]. Moreover, it appears that the strong internal magnetic field should break the channel symmetry and consequently destroy the non-Fermi liquid behaviour.

Recently a square-root-of- $T$ increase of the electrical resistivity was found for a cluster-glass compound $\mathrm{U}_{2} \mathrm{CoSi}_{3}$ that is isostructural to $\mathrm{U}_{2} \mathrm{NiSi}_{3}$ [12]. The overall temperature and magnetic field dependences of the electrical transport in this material were successfully interpreted by taking into account quantum interference effects, which result from distinct crystallographic disorder. Taking into account the large values of the residual resistivity and the small values of RRR observed for single-crystalline $\mathrm{U}_{2} \mathrm{NiSi}_{3}$ it seems likely that similar scenario is applicable in the present case. While the electron-electron interaction manifests itself as a $T^{0.5}$ increase in the in-plane and out-of-plane electrical resistivity below $5 \mathrm{~K}$, the weak localization effect is observed solely in the $a b$-plane resistivity as a linear-in- $T$ contribution to $\rho_{a b}(T) / \rho_{a b}(2 \mathrm{~K})$. It implies that internal magnetic field due to exchange interaction does not break the interference of closed trajectories of electrons moving in the $a b$-plane in opposite directions, similarly to the case of $2 \mathrm{D}$ disordered ferromagnets with in-plane magnetic induction [13]. Under hydrostatic pressure one observes an increase of the normal transport resistivity contribution with respect to the quantum correction contribution, which is evidenced by the shift of the resistivity minimum to lower temperatures and by the increase of RRR. As a result, the weak localization effect in the $a b$-plane resistivity gets suppressed. Surprisingly high pressure hardly affects the electron-electron interaction in the $a b$-plane resistivity, and the slope of the $T^{0.5}$ upturn increases. Since the diffusion coefficient and the electron-electron interaction constant are directly dependent on the Fermi surface properties, it seems likely that the observed behaviour originates from pressure-induced changes in the electronic structure of $\mathrm{U}_{2} \mathrm{NiSi}_{3}$.

\section{Conclusions}

The low-temperature electrical resistivity of $\mathrm{U}_{2} \mathrm{NiSi}_{3}$ seems governed by the interplay of ferromagnetism and quantum interference effects. The resistivity increase proportional to $T^{0.5}$, observed below $5 \mathrm{~K}$, probably results from the electron-electron interaction, and is not destroyed by hydrostatic pressure as high as 2.4 GPa. On the other hand, the weak localization correction, observed below $T_{\mathrm{C}}$ solely in the $a b$-plane resistivity, gets suppressed under high pressure. This finding suggests that pressure-controlled changes in the electronic structure of $\mathrm{U}_{2} \mathrm{NiSi}_{3}$ may have a profound influence on the quantum interference effects.

\section{Acknowledgments}

We are grateful to Dr. A. Gągor for orienting the single crystals on a four-circle diffractometer. This work was supported by the National Science Centre (Poland) under grants 2011/03/D/ST3/02351 and 2011/01/B/ST3/04482.

\section{References}

[1] E. Abrahams, P.W. Anderson, D.C. Licciardello, T.V. Ramakrishnan, Phys. Rev. Lett. 42, 673 (1979).

[2] B.L. Altshuler, A.G. Aronov, Sov. Phys. JETP 50, 968 (1979).

[3] P.A. Lee, T.V. Ramakrishnan, Rev. Mod. Phys. 57, 287 (1985).

[4] B.L. Altshuler, A.G. Aronov, Electron-Electron Interactions in Disordered Systems, Elsevier, Amsterdam 1985.

[5] M. Szlawska, D. Kaczorowski, M. Reehuis, Phys. Rev. B 81, 094423 (2010).

[6] M. Szlawska, Ph.D. Thesis, INTiBS PAN, Wrocław 2011.

[7] D. Li, A. Dönni, Y. Kimura, Y. Shiokawa, Y. Homma, Y. Haga, E. Yamamoto, T. Honma, Y. Onuki, J. Phys. Condens. Matter 11, 8263 (1999).

[8] D. Kaczorowski, H. Noël , J. Phys. Condens. Matter 5, 9185 (1993).

[9] D.B. Chevalier, R. Pöttgen, B. Darriet, P. Gravereau, J. Etourneau, J. Alloys Comp. 233, 150 (1996).

[10] D.A. Schröder, M.F. Collins, C.V. Stager, J.D. Garrett, J.E. Greedan, Z. Tun, A.O. dos Santos, A.N. Medina, J. Magn. Magn. Mater. 140-144, 1407 (1995).

[11] I.L. Aleiner, B.L. Altshuler, Y.M. Galperin, T.A. Shutenko, Phys. Rev. Lett. 86, 2629 (2001).

[12] M. Szlawska, D. Gnida, D. Kaczorowski, Phys. Rev. B 84, 134410 (2011).

[13] V. Dugaev, P. Bruno, J. Barnaś, Phys. Rev. B 64, 144423 (2001). 